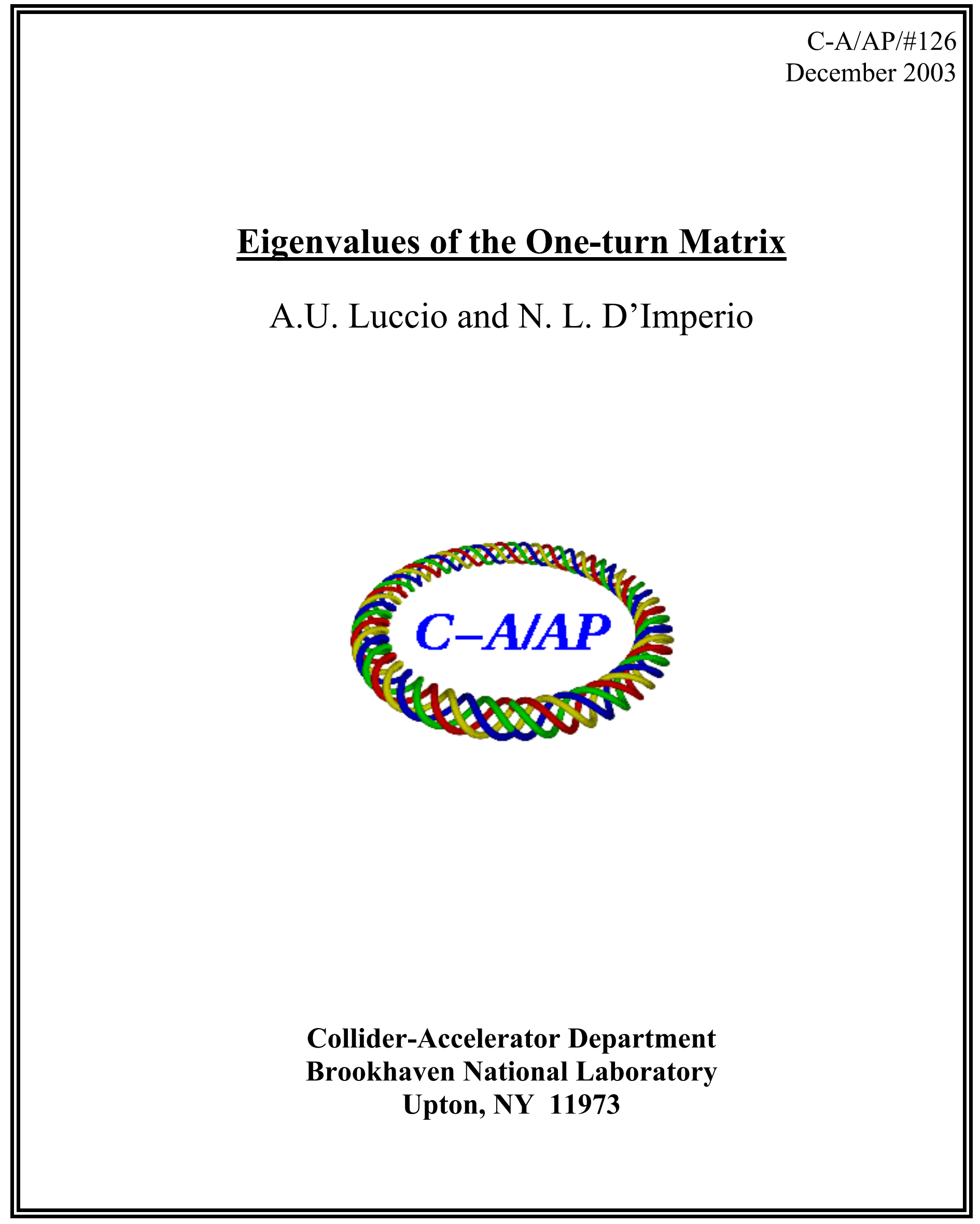




\title{
Eigenvalues of the One-turn Matrix
}

\author{
A.U. Luccio and N.L.D'Imperio
}

December 4, 2003

\section{Betatron Tune and Twiss Functions from the OTM}

In PIC tracking in a circular accelerator, the betatron tune of a particle in the herd is often calculated by performing an FFT analysis of its orbit or from a running average of its transverse oscillations wavelength. With both techniqes, the tune will be only known after a considerable number of turns. It is often desirable to calculate a quasi instantaneous value of the betatron tunes of a particle in order to study how the distribution of tunes in the beam evolves. This can be done using the One Turn Matrix (OTM) associated with each particle in the herd. The algorithm described in the following is being implemented in the tracking code $S I M B A D$, a remastering of the Brookhaven-Oak Ridge code ORBIT [1]

The 36 elements of a $6 \times 6$ OTM in phase space can be calculated by solving 36 linear equations between the coordinates of a particle in 5 successive turns, from turn $\mathrm{N}$ to turn $\mathrm{N}+5$

$$
x_{i}^{(n+1)}=\sum_{j=1}^{6} M_{i, j} x_{j}^{(n)}, \quad i=1,6, \quad n=N, N+5,
$$

with $n=$ turn number, and $x_{i} \equiv\left(x, p_{x}, y, p_{y}, \phi, \Delta p / p\right)$.

The particle OTM of a circular accelerator, in one of the transverse dimensions can be written as

$$
M^{(2)}=\left(\begin{array}{cc}
C+\alpha S & \beta S \\
-\gamma S & C-\alpha S
\end{array}\right)
$$

where

$$
C=\cos (2 \pi \nu), \quad S=\sin (2 \pi \nu),
$$

with $\tilde{\nu}$ the (fractional part of the) betatron tune $\nu$ and $\alpha, \beta, \gamma$ the Courant-Snyder coefficients. It is

$$
\tilde{\nu}=\frac{1}{2 \pi} \arccos \left(\frac{\operatorname{Tr}(M)}{2}\right) \text {. }
$$

The arccos function has two values between 0 and $2 \pi$, i.e. $\theta$ and $2 \pi-\theta$. Which one one should choose? In the $2 \times 2$ case -and, actually, in the decoupled case that we will examine next- the answer comes from inspection of the $M_{1,2}$ element of the matrix. Since $\beta$ must be positive by definition, the right solution is for $S$ to be of the same sign of $M_{1,2}$.

Since it is $\beta \gamma=1+\alpha^{2}$, the characteristic equation $\operatorname{det}(M-\lambda)=0$ for the matrix of Eq.(2) can be written as

$$
\operatorname{det}(M)=\lambda^{2}-2 C \lambda+1=0
$$

with roots (eigenvalues)

$$
\lambda_{1}=C+i S, \quad \lambda_{2}=C-i S .
$$

Then the tune can also be calculated -other than by Eq.(3)- from the arctan of the ratio of the imaginary part to the real part of the eigenvalues

$$
\tilde{\nu}=\frac{1}{2 \pi} \arctan \left(\frac{\operatorname{Im}(\lambda)}{\operatorname{Re}(\lambda)}\right)=\frac{1}{2 \pi} \arctan \left(\frac{S}{C}\right) .
$$


Consider now a $4 \times 4$ matrix for the transverse motion with no coupling between $x$ and $y$

$$
M^{(4)}=\left(\begin{array}{cccc}
C_{x}+\alpha_{x} S_{x} & \beta_{x} S_{x} & 0 & 0 \\
-\gamma_{x} S_{x} & C_{x}-\alpha_{x} S_{x} & 0 & 0 \\
0 & 0 & C_{y}+\alpha_{y} S_{y} & \beta_{y} S_{y} \\
0 & 0 & -\gamma_{y} S_{y} & C_{y}-\alpha_{y} S_{y}
\end{array}\right)
$$

The characteristic equation is

$$
\left(\lambda^{2}-2 C_{x} \lambda+1\right)\left(\lambda^{2}-2 C_{y} \lambda+1\right)=0
$$

with eigenvalues

$$
\lambda_{1}=C_{x}+i S_{x}, \quad \lambda_{2}=C_{x}-i S_{x}, \quad \lambda_{3}=C_{y}+i S_{y}, \quad \lambda_{4}=C_{y}-i S_{y},
$$

so, the tunes can be calculated separately from the traces of the respective $2 \times 2$ sub-matrices, or from the eigenvalues.

From the OTM we can also calculate the twiss functions $\alpha$ and $\beta$. For each transverse dimension and no coupling, from Eq. (2) obtain

$$
\alpha=\frac{M_{1,1}-M_{2,2}}{2 \operatorname{Im}(\lambda)}, \quad \beta=\frac{M_{1,2}}{2 \operatorname{Im}(\lambda)}, \quad(\text { for } \mathrm{x} ; 3 \text { and } 4 \text { for } \mathrm{y} \text { ). }
$$

We will use the values of $\operatorname{Im}(\lambda)$ with the same sign of $M_{1,2}$ in order to obtain a positive value of $\beta$.

In general, we will consider the full $6 \times 6$ One Turn Matrix. This is particularly important in the presence of coupling and dispersion.

\section{Recipe}

In practice, tune calculation using the OTM is only efficient if the particle betatron tune is not too different than the bare tune. The algorithm can be best implemented in a post processor for any tracking program whose output is the complete phase space of the particle at each turn. The recipe follows the steps:

1. Construct the $6 \times 6$ One Turn Matrix from a solution of the system of 36 linear equations of Eq.(1), using the phase space coordinates of the particle over 5 turns.

2. Find the 6 conjugate complex eigenvalues of the OTM with an eigenvalue solver, like EISPACK.

3. Calculate $\alpha$ and $\beta$ using Eq. (5). Select the eigenvalues with the imaginary part of the same sign of $M_{1,2}$ or $M_{3,4}$ (to make $\beta>1$ ).

4. Compare $\alpha$ and $\beta$ with the bare lattice values. This is the best way to tell which eigenvalues are for $x$ and which for $y$. For best results, let us calculate the OTM in a location in the lattice where the two values of $\alpha$ and $\beta$ for the bare lattice differ the most.

5. Calculate the fractional part of the tune $\tilde{\nu}$ using Eq. (4). If the sign is negative, 1 must be added (corresponding to two possible value of the arctan).

\section{Example: RHIC}

For $S I M B A D$ low intensity proton tracking in a standard $M A D$ [2] lattice of RHIC, the OTM of a particle taken at random in the herd was

$$
\left(\begin{array}{cccccc}
-0.47535947 & 8.53011131 & -0.00589695 & -0.19126983 & 0 . & -0.03032236 \\
-0.08754899 & -0.53383231 & -0.00262825 & 0.01054437 & 0 . & 0.00360564 \\
0.02068402 & -0.16990490 & 0.71568137 & 6.98957968 & 0 . & 0.01341598 \\
-0.00098221 & -0.01924209 & -0.06942750 & 0.72000730 & 0 . & 0.00336203 \\
0.00428595 & -0.01425635 & -0.00323663 & -0.01346969 & 1 . & -7.23540640 \\
0 . & 0 . & 0 . & 0 . & 0 . & 1 .
\end{array}\right) .
$$


Table 1: AGS parameters for PIC simulation

\begin{tabular}{ll}
\hline $2 \pi R_{s}$ & $807.076 \mathrm{~m}$ \\
Kinetic Energy & $1.2 \mathrm{GeV}$ \\
no of PIC macros & $10^{5}$ \\
beam profile, random & Gaussian (Trans.) white (Long.) \\
emittances $\epsilon_{x}, \epsilon_{y}$ & $40,10 \mathrm{~mm}-\mathrm{mrad}$ \\
r.m.s. $\Delta E / E$ & $1 \times 10^{-3}$ \\
\hline
\end{tabular}

The non vanishing matrix elements $M_{1,3}, M_{1,4}, M_{3,1}, M_{3,2}, M_{4,1}, M_{4,2}$ show the presence of coupling, due to the sextupoles in the lattice. The elements $M_{i, 6}, i=1,5$ and $M_{5, j}, j=1,4$ contain the dispersion. Following Eq.(4) the decoupled tune can be calculated from the trace of the $2 \times 2$ transverse sub matrices

$$
\begin{array}{ccc}
\text { Partial Traces : } & -1.00919175 & 1.43568873 \\
\text { Decoupled Tunes : } & 0.334179014 & 0.122564375
\end{array}
$$

Let us now calculate the eigenvalues of the full matrix with EISPACK and obtain, with: $R=$ Real part of the eigenvalues and $I=$ Imaginary part

$$
\begin{array}{ccccccc}
\mathrm{R}: & -0.504428089 & -0.504428089 & 1 . & 0.71767652 & 0.71767652 & 1 . \\
\mathrm{I}: & -0.863453805 & 0.863453805 & 0 . & -0.696376383 & 0.696376383 & 0 . \\
\arctan (I / R) / 2 \pi: & -0.334148049 & 0.334148049 & 0 . & -0.122602709 & 0.122602709 & 0 .
\end{array}
$$

Compare the values calculated from the eigenvalues with the values calculated by $M A D$ for a zero amplitude closed orbit and $\Delta p / p=0^{1}$

$$
\begin{array}{ccccccc} 
& \tilde{\nu} & \alpha_{x} & \beta_{x} & \tilde{\nu} & \alpha_{y} & \beta_{y} \\
& 0.33414811 & 0.03385986 & 9.87905884 & 0.12260274 & -0.00310603 & 10.03707120 \\
M A D & {[28] .33418915} & 0.03405129 & 9.87723727 & {[29] .12259701} & -0.00309503 & 10.03222775
\end{array}
$$

Other particles in the herd will have slighly different tunes and values of Twiss parameters.

\section{Four examples: AGS}

We examined a configuration of the AGS with a coasting (long) beam and with short beam bunches and a high RF harmonic, at constant enegy -no acceleration- AGS and simulation parameters common to all cases are shown in Table 1. Main motivation was to study the AGS as a possible high intensity proton driver for a neutrino factory. We wanted to compare cases with low and high space charge and with long and short bunches.

In the chart of tune distribution in the beam (tune footprint), we put for comparison the bare tune of the lattice and the shifted tune calculated with the classical formula (Laslett) for tune shift due to space charge in a beam with Gaussian round transverse density

$$
\Delta \nu=-\frac{r_{0}}{(2 \pi)^{3 / 2}} \frac{N}{\varepsilon \beta^{2} \gamma^{3} B_{f}}
$$

with $r_{0}=1.5410^{-18}$ the classical electron radius, $N$ the number of particles in the bunch, $\varepsilon$ the r.m.s. emittance of a Gaussian beam, and $B_{f}$ is the bunching factor defined as

$$
B_{f}=\frac{I}{I_{p e a k}}=\frac{\delta \phi}{2 \pi}
$$

with $I$ the total beam current and $\delta \phi$ the bunch r.m.s. length in radians.

\footnotetext{
${ }^{1}$ The integer part of the $M A D$ calculated tune is shown in [ ]
} 


\subsection{Propagation at low intensity}

This is an example of SIMBAD proton tracking in the AGS with very little space charge effects. Beam charge was $10^{9} \mathrm{~A}$-s. In this case there are no lattice coupling elements and no vertical bends, so dispersion only acts between $x, x^{\prime}$ and $\Delta E / E$ and between $\phi$ and $x, x^{\prime}$. The OTM for a random para axial particle with $\Delta E / E=0$ resulted

$$
\left(\begin{array}{cccccc}
0.86589497 & -14.43480015 & 0 . & 0 . & 0 . & 2.66993999 \\
0.16401200 & -1.57927001 & 0 . & 0 . & 0 . & 0.09289760 \\
0 . & 0 . & -1.39865005 & -15.29469967 & 0 . & 0 . \\
0 . & 0 . & 0.17456800 & 1.19398999 & 0 . & 0 . \\
0.35746199 & -2.87561011 & 0 . & 0 . & 1 . & 178.1499939 \\
0 . & 0 . & 0 . & 0 . & 0 . & 1 .
\end{array}\right) .
$$

with eigenvalues

$$
\begin{array}{ccccccc}
\mathrm{R}: & -0.356687725 & -0.356687725 & 1.00000024 & -0.102330089 & -0.102330089 & 1 \\
\mathrm{I}: & -0.934223056 & 0.934223056 & 0 . & -0.99474597 & 0.99474597 & 0 .
\end{array} .
$$

Since both $M_{1,2}$ and $M_{3,4}$ are negative, for a positive $\beta$, according to Eq.(5) choose the two negative imaginary eigenvalue, respectively. With these, the tune fractional parts are (note the "1-" that is approriate when the fractional part of the tune would appear negative from this calculation)

$$
\arctan (I / R) / 2 \pi: \quad 1-0.308046818=0.691953, \quad 1-0.266314775=0.733685 .
$$

The eigenvalues describe the oscillation modes represented in the OTM. Let us find $\alpha$ and $\beta$, then following step 4 of our Recipe we can correctly attribute the calculated values of the tunes to the transverse and to the vertical mode, respectively. We see which eigenvalue (1) corresponds to the $x$ motion and which (4) to $y$. Find in good agreement with $M A D^{2}$

$\begin{array}{ccccccc} & \tilde{\nu_{x}} & \alpha_{x} & \beta_{x} & \tilde{\nu_{y}} & \alpha_{y} & \beta_{y} \\ & 0.691953 & -1.30866 & 15.4511 & 0.733685 & 1.30317 & 15.3755 \\ M A D & {[8] .69195} & -1.30867 & 15.4512 & {[8] .73368} & 1.30315 & 15.3754\end{array}$

\subsection{Coasting beam with high space charge}

We tracked with SIMBAD an intense coasting beam of protons in the AGS. Beam charge was $4.10^{14}$ A-s, bunch length $360 \mathrm{deg}$. The 3D calculation of the space charge force was an important part of the exercise: space charge also produces coupling between the oscillation modes. The OTM for a random particle in the herd was in this case

$$
\left(\begin{array}{rrrrrr}
-0.87052 & -18.603 & -3.6017[-02] & 5.0023[-01] & 9.3295[-01] & 4.3267[+04] \\
4.2577[-02] & -0.21484 & -2.3785[-03] & -1.0979[-02] & -3.6528[-02] & -1.7517[+03] \\
-1.7346[-02] & -2.1124[-01] & -0.19359 & -9.3604 & 1.3638[+00] & 6.0800[+04] \\
-8.9606[-05] & -2.3363[-03] & 8.9356[-02] & -0.61184 & 6.1254[-02] & 2.7182[+03] \\
-6.1469[-09] & -1.0726[-07] & 2.4514[-09] & -1.0521[-07] & 1 & -6.3814[-01] \\
-2.4426[-08] & 2.2002[-07] & -1.0332[-08] & -4.0270[-07] & 3.6691[-07] & 1.0463
\end{array}\right),
$$

with eigenvalues

$$
\begin{array}{ccccccc}
\mathrm{R}: & -0.54568 & -0.54568 & -0.39857 & -0.39857 & 1.0440 & 1.0000 \\
\mathrm{I}: & -0.82715 & 0.82715 & -0.89033 & 0.89033 & 0.0000 & 0.0000
\end{array}
$$

In this case, the selection is for the 2.nd and 4.th eigenvalues in the table. Tunes and Twiss functions are

$$
\begin{array}{ccccccc} 
& \tilde{\nu_{x}} & \alpha_{x} & \beta_{x} & \tilde{\nu_{y}} & \alpha_{y} & \beta_{y} \\
& 0.65719 & 0.39635 & 22.491 & 0.68301 & -0.23489 & 10.5135 \\
M A D & {[8] .69195} & 0.38461 & 22.407 & {[8] .73368} & -0.23583 & 10.3021
\end{array}
$$

\footnotetext{
${ }^{2}$ In this lattice location, the values of $\beta$ for the two planes were close, however the values of $\alpha$ were wide different. The choice between $x$ and $y$ was clear
} 


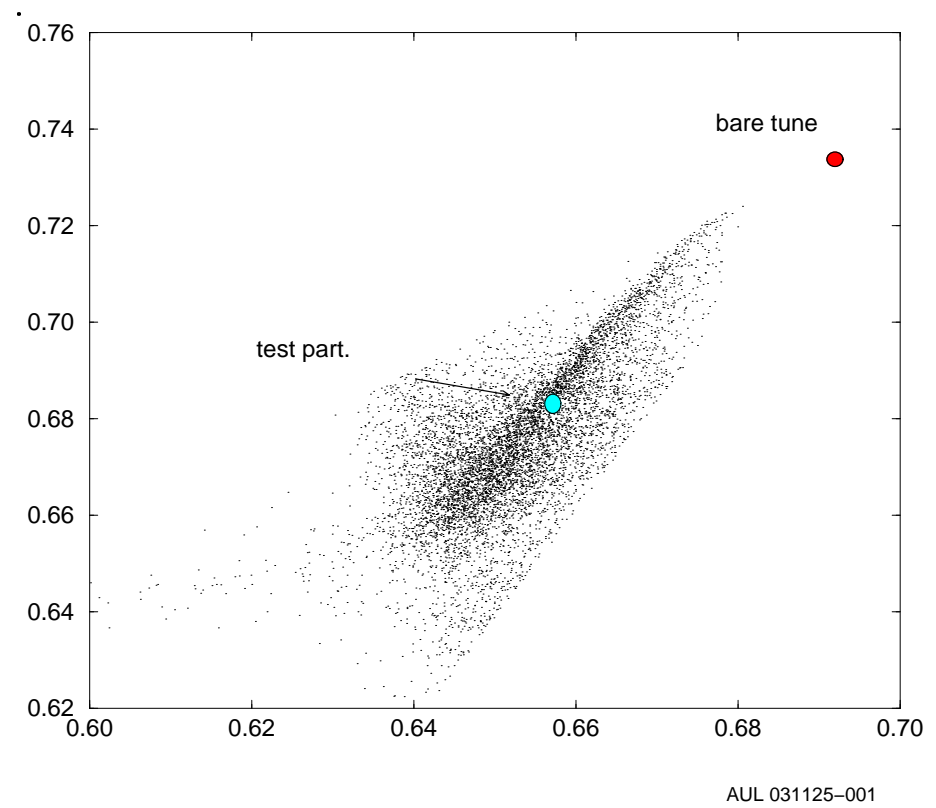

Figure 1: Tune footprint for a high intensity proton coasting beam in the AGS. The plot contains over $80 \%$ of the tune points. The bare tune, as well as the tune of a particle taken as example are shown

Table 2: AGS. Short bunches

\begin{tabular}{ll}
\hline bunch charge & $4.10^{12}$ \\
bunch length $\Delta \phi$ & $10 \mathrm{deg}$ \\
RF harmonic & 24 \\
RF voltage & $800 \mathrm{KV}$ \\
\hline
\end{tabular}

The tune depression for this particle, due to space charge, over 5 turns is

$$
\Delta \nu \quad-0.03476 \quad-0.05067
$$

The tune footprint for over $80 \%$ of the particles in the herd is shown if Fig.1. The locations of the bare tune from $M A D$ and of the tune used in the example above are shown. The tunes of the remaining particles are scattered all around.

\subsection{Short bunches beam with high space charge. Separate betatron tunes}

For this case we used a high RF harmonic. Parameters for the simulation are in table 2. Bare lattice betatron tunes were kept widely apart. For a specific particle, we obtained the following OTM

$$
\left(\begin{array}{rrrrrr}
0.22121] & -18.475 & -4.5768[-03] & -9.0866[-02] & -2.6632 & 3.7222[+02] \\
4.3991[-02] & 0.75997 & 4.2703[-05] & 4.7763[-03] & -0.25451 & -4.3409[+01] \\
1.8114[-03] & 4.2767[-02] & -0.95111 & -1.7633 & -5.5742 & 1.7401[+02] \\
6.9382[-04] & -1.1476[-02] & 1.5836[-02] & -1.0219 & -8.2160[-02] & 3.6929 \\
1.6566[-10] & 1.5966[-08] & 1.4420[-09] & 6.1351[-08] & 1.0000 & -0.65159 \\
-3.9621[-07] & -3.2822[-06] & -1.6053[-07] & 1.7935[-06] & 1.1519[-02] & 1.0160
\end{array}\right),
$$

with eigenvalues

$$
\begin{array}{ccccccc}
\mathrm{R}: & 0.49071 & 0.49071 & -0.98646 & -0.98646 & 1.0078 & 1.0078 \\
\mathrm{I}: & -0.86026 & 0.86026 & -0.16335 & 0.16335 & -0.086292 & 0.086292
\end{array}
$$




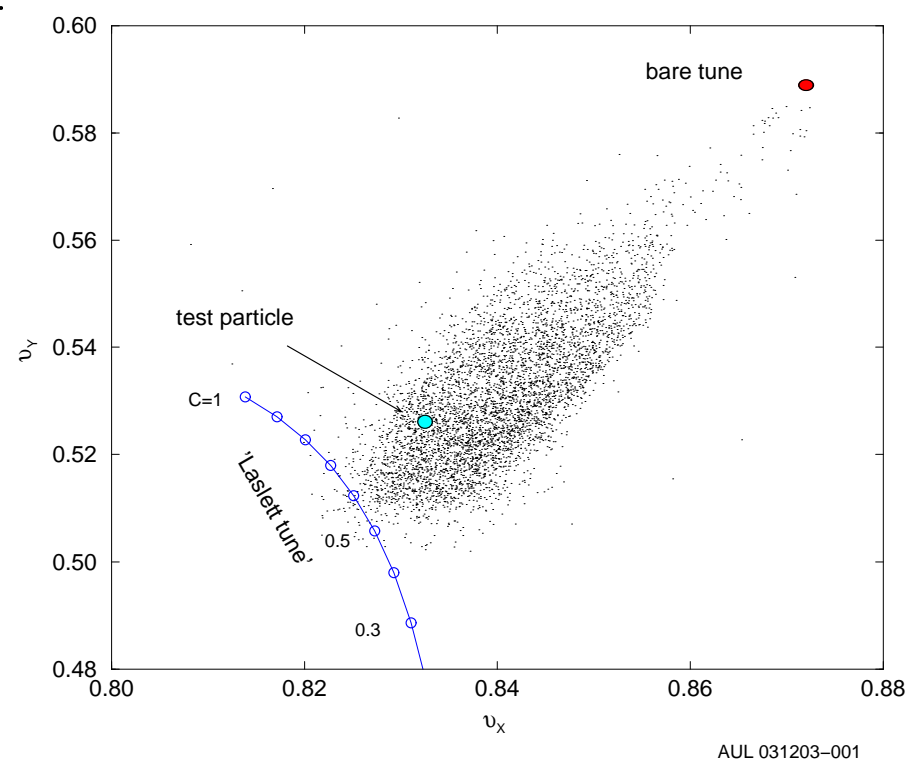

Figure 2: Tune footprint for a high intensity short beam in the AGS. The bare tune, the tune of a particle taken as example, and the 'Laslett' tunes, as a function of coupling $C$ are shown

In this case, the selection is for eigenvalues \#1 and \#3. The tunes and the Twiss functions are

$$
\begin{array}{ccccccc} 
& \tilde{\nu_{x}} & \alpha_{x} & \beta_{x} & \tilde{\nu_{y}} & \alpha_{y} & \beta_{y} \\
& 0.8325 & 0.313 & 21.476 & 0.5261 & -0.2166 & 10.795 \\
M A D & {[8] .8720} & 0.332 & 21.462 & {[8] .5889} & -0.2081 & 10.672
\end{array}
$$

The tune depression for this particle, due to space charge, over 5 turns is

$$
\Delta \nu \quad-0.0395 \quad-0.0628
$$

The betatron tune footprint is shown if Fig. 2. The locations of the bare tune from MAD and of the test particle are shown. The theoretical depressed tune, due to space charge and coupling in the transverse space can be calculated from Eq.(6). Since the formula is valid only for a round beam, we obtained the $x-$ and $-y$ depression defining an effective emittance in each direction renormalized as follows

$$
\begin{gathered}
\varepsilon_{x}=(1-C) \varepsilon_{x}^{(0)}+C<\varepsilon>, \quad \varepsilon_{y}=(1-C) \varepsilon_{y}^{(0)}+C<\varepsilon> \\
<\varepsilon>=\left(\varepsilon_{x}^{(0)}+\varepsilon_{y}^{(0)}\right) / 2
\end{gathered}
$$

with $\langle\varepsilon>$ the average of the two emittances and $C$ a coupling coefficient. The figure shows that the coupling, due mainly to space charge, is between 0.5 and 0.6 for this example.

\subsection{Short bunch. Close betatron tunes. Octupole Resonance}

For this example [3] parameters were as in the preceding section, but with betatron tunes closer together. The case is particuarly interesting because it shows the behaviour of the beam crossing an octupole resonance.

The tune footprint shows in this case two isolated regions, corresponding to particles residing in the core of the beam and in the halo, respectively. A tune footprint pattern pattern rapidly forms and establishes itself during the synchrotron motion. Fig. 3 shows the tunes of 10,000 out of the 100,000 tracked, at a certain turn (turn 24). In the betatron tune space two islands appear separated by the line $2 \tilde{\nu}_{x}=2 \tilde{\nu}_{y}$. The average of the fractional part of the tune in the upper island and the lower island, compared with the bare tune of the lattice are as follows -the table also shows how 


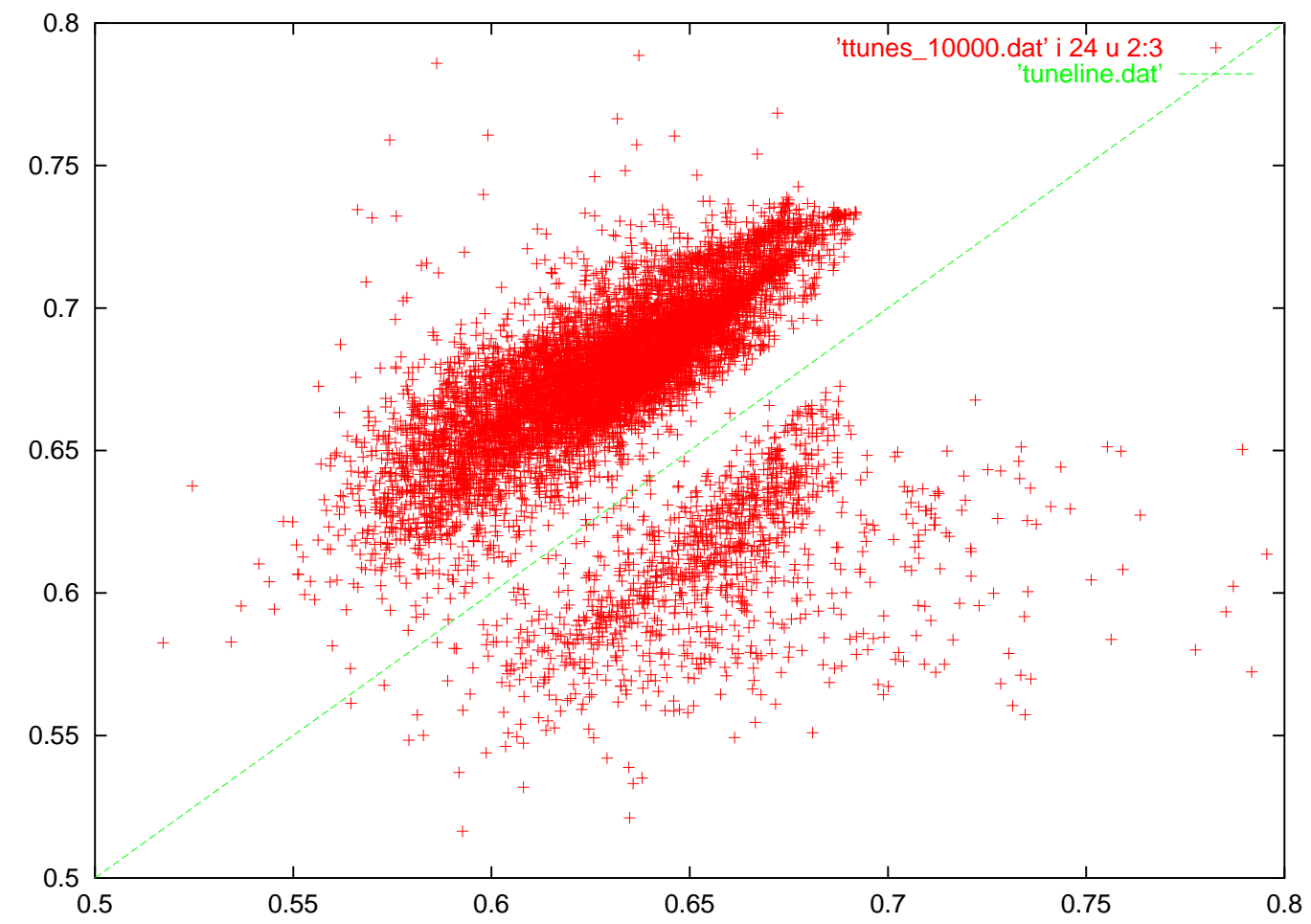

Figure 3: High intensity. Turn 24. Two islands of betatron tune show up. The instability line $2 \nu_{x}-2 \nu_{y}$ is also shown.

many macros fall in each island-

$\begin{array}{cccccc} & \tilde{\nu}_{x} & \tilde{\nu}_{y} & \delta \nu_{x} & \delta \nu_{y} & n_{\text {macro }} \\ \text { bare tune } & 0.69195 & 0.73368 & & & \\ \text { upper island } & 0.63073 & 0.67956 & -0.06122 & -0.05412 & 8156 \\ \text { lower island } & 0.65343 & 0.61105 & & & 995\end{array}$

The number of macros (out of 10,000) in either island is also shown. The rest is scattered around. Including all particles, the values for the average tunes are 0.61990/0.68032 (8575 macros above the line) and $0.65285 / 0.55040$ (1425 macros below the line). Similarly, in Fig. 4 we show the values of the envelope functions $\beta_{x}$ and $\beta_{y}$ for the two groups

$\begin{array}{ccccc} & \beta_{x} & \beta_{y} & \alpha_{x} & \alpha_{y} \\ \text { bare Twiss } & 22.407 & 10.3021 & 0.38461 & -0.23583 \\ \text { upper island } & 21.7766 & 10.5865 & & \\ \text { lower island } & 19.0730 & 17.0056 & & \end{array}$

\section{References}

[1] J.D.Galambos, J.A.Holmes, D.K.Olsen A.Luccio and J.Beebe-Wang: Orbit User's Manual Vers. 1.10. Technical Report SNS/ORNL/AP 011, Rev.1, 1999.

[2] H.Grote and F.Ch.IsElin: The MAD program, Vers.8.19. Technical Report CERN/SL/90-13, Geneva, Switzerland, 1996.

[3] A.U.Luccio and N.L.D'ImPerio: Simulation of the AGS as a Proton Driver. In: ICFA Beam Dynamics Mini Workshop on Space Charge Simulation, Oxford, UK, Trinity College, April 2-4, 2003, www-bd.fnal/icfa/workshops, 2003. 


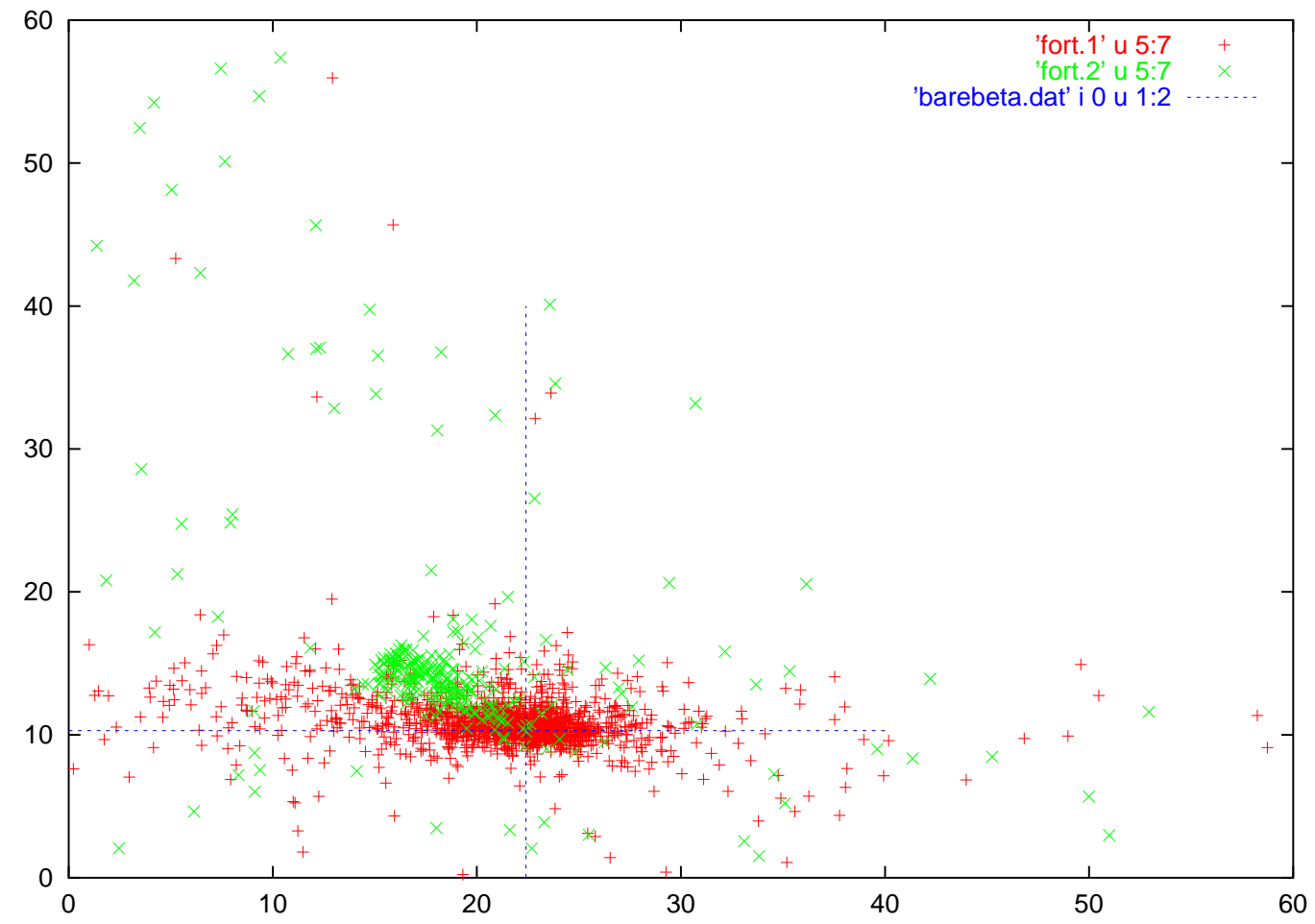

Figure 4: High intensity. Turn 24. Two islands of envelope function $\beta_{y}$ vs. $\beta_{x}$. The location of the bare lattice $\beta$ is also shown. 